\title{
Fascin expression predicts an aggressive clinical course in patients with advanced breast cancer
}

\author{
KYUENG-WHAN MIN ${ }^{1}$, SEOUNG WAN CHAE ${ }^{2 *}$, DONG-HOON KIM ${ }^{2 *}$, SUNG-IM DO ${ }^{2}$, \\ KYUNGEUN KIM ${ }^{2}$, HYUN JOO LEE ${ }^{2}$, JIN HEE SOHN ${ }^{2}$, JUNG-SOO PYO ${ }^{2}$, DONG HYUN KIM ${ }^{2}$, \\ SUKJOONG $\mathrm{OH}^{3}$, SEON HYEONG $\mathrm{CHOI}^{4}$, YONG LAI PARK ${ }^{5}$ and CHAN HEUN PARK ${ }^{5}$
}

\begin{abstract}
${ }^{1}$ Department of Pathology, Hallym University Sacred Heart Hospital, Hallym University College of Medicine, Anyang, Gyeonggi-do; Departments of ${ }^{2}$ Pathology, ${ }^{3}$ Internal Medicine, ${ }^{4}$ Radiology and ${ }^{5}$ Surgery, Kangbuk Samsung Hospital, Sungkyunkwan University School of Medicine, Seoul, Republic of Korea
\end{abstract}

Received September 28, 2014; Accepted April 20, 2015

DOI: $10.3892 / 01.2015 .3191$

\begin{abstract}
Fascin is an actin cross-linking protein, which regulates actin dynamics and filopodia or spike formation, as well as the epithelial-mesenchymal transition, and has been implicated in cell motility. Although, fascin is pivotal in mediating the aggressive behaviour of various types of cancer, its prognostic significance according to tumour stage has yet to be evaluated. Therefore, the present study investigated fascin expression in 194 patients diagnosed with invasive ductal carcinoma of the breast between 2000 and 2005. Fascin protein expression levels were evaluated by immunostaining on a tissue microarray, and the association between fascin expression and various clinicopathological parameters was analysed. Fascin expression was significantly correlated with various clinicopathological parameters, including high histological grade, tumour necrosis, resistance to adjuvant therapy, high expression of p53 and Ki-67 and specific therapeutic markers (oestrogen and progesterone receptor negativity; all $\mathrm{P}<0.05$ ). Furthermore, univariate and multivariate analyses identified a significant association between fascin expression, and poor disease-free and overall survival, in late-stage breast cancer (all $\mathrm{P}<0.05$ ). Therefore, fascin may be crucial in predicting aggressive tumour behaviour, particularly in patients with advanced-stage disease that has acquired the properties of migration and invasion.
\end{abstract}

Correspondence to: Professor Seoung Wan Chae or Professor Dong-Hoon Kim, Department of Pathology, Kangbuk Samsung Hospital, Sungkyunkwan University School of Medicine, 29 Saemunan-ro, Seoul 110-746, Republic of Korea

E-mail: swan.chae@samsung.com

E-mail: idavid.kim@samsung.com

*Contributed equally

Key words: fascin-1 protein, prognosis, invasive ductal carcinoma, human breast cancer

\section{Introduction}

Breast cancer is the most commonly diagnosed neoplasm and the third leading cause of cancer-associated mortality in the United States, with 22.2 mortalities per 100,000 women associated with breast cancer each year. The five-year relative survival rate for breast cancer has gradually increased since the early 1990s and between 2007 and 2011 it was $~ 89.2 \%$. However, the prognosis of patients with breast cancer is dependent on the disease stage at the time of diagnosis. In particular, the survival rates of patients with localised disease and regional lymph node metastasis at diagnosis are higher than those of patients presenting with distant metastasis (1). A number of studies have established molecular markers, which are associated with distinct histopathological features, the response to adjuvant therapy and/or the clinical outcome of breast cancer (2-7). Furthermore, the following clinicopathological factors are considered to be useful markers for predicting prognosis and identifying therapeutic targets in patients with advanced breast cancer: American Joint Committee on Cancer (AJCC) stage, histological grade, oestrogen receptor (ER) and progesterone receptor (PR) expression, human epithelial growth factor receptor 2 (HER2) amplification, p53 expression and Ki-67 labelling index (2-6). Based on data obtained from molecular or immunohistochemical (IHC) analyses, breast cancer is classified into four major subtypes: Luminal A, luminal B, basal-like and HER2-positive (7). More recently, the luminal B subtype has been subdivided according to HER2 status and Ki-67 labelling index (8).

Despite improvements in the treatment of breast cancer, the high mortality rate of patients with direct invasion of adjacent organs or distant metastases remains a problem (9). Therefore, improved understanding of the molecular and cellular mechanisms of tumour invasion and metastasis is required for the development of more effective treatment strategies. The multi-step process of metastasis involves numerous cellular events, including neovascularisation, stromal invasion, lymphovascular invasion and growth at a secondary site $(10,11)$. Furthermore, increased tumour cell motility, combined with extracellular matrix degradation at the invasive front of the tumour, are critical early processes in metastasis. (12) 
Fascin-1 is a 55-kDa cytoskeletal actin-binding protein that packages actin filaments into tertiary structures, including microspikes, stress fibres and membrane ruffles, within dynamic cellular structures, resulting in the enhancement of cell motility, migration and adhesion $(13,14)$. Fascin-1 (also known as fascin) is primarily expressed during embryonic development, while its expression in adults is highly restricted to neurons, glial cells, endothelial cells and antigen-presenting dendritic cells (15). The additional forms of fascin, fascin-2 and -3 , are expressed in retinal photoreceptor cells and the testes, respectively (16). Published data has demonstrated that fascin is upregulated or highly expressed in the human cancer of various organs, including the oesophagus (17), breast (18), colon (19), lung (20), stomach (21) and urinary bladder (22), as well as in individual tissues, and that the expression of fascin is associated with aggressive behaviour (23). Previous studies of breast cancer have identified that fascin overexpression is associated with factors representing aggressive tumor behaviour, for example hormonal receptor negativity, a triple-negative subtype and/or a basal-like phenotype (24-26). However, to the best of our knowledge, no reports have thus far identified a correlation between fascin expression and disease-free or overall survival rates, according to the AJCC tumor node metastasis (TNM) stage.

Therefore, the aim of the present study was to investigate fascin expression in a large cohort of patients with invasive ductal carcinoma (IDC) of the breast, and to assess any statistical correlations between fascin expression, and clinicopathologic parameters, molecular subtypes and patient survival according to the AJCC stage of breast cancer.

\section{Patients and methods}

Patient selection. The present study included 194 Korean women diagnosed with IDC at Kangbuk Samsung Hospital, Sungkyunkwan University School of Medicine (Seoul, Republic of Korea) between 2000 and 2005. Various clinicopathological variables were established by reviewing patient records and haematoxylin and eosin-stained slides. For example, histological grade was determined using the modified Bloom-Richardson-Elston grading system (27). Additionally, tumours were staged with reference to the size and/or extent of the tumour, regional lymph node involvement and metastasis, using the seventh edition of the AJCC TNM classification system (28).

Ethical Statement. The study was performed according to the Declaration of Helsinki and approved by the local Ethics Committee of the Kangbuk Samsung Hospital (KBSMC14011, Sungkyunkwan University School of Medicine, Seoul, Republic of Korea).

Tissue microarray (TMA) construction. A series of 194 tumour TMA specimens were assembled using a tissue-array instrument (AccuMax ${ }^{\mathrm{TM}}$ Array; ISU ABXIS Co. Ltd., Seoul, Korea). The TMAs consisted of 10x6 arrays of IDC tissue cores measuring 2.0- $\mathrm{mm}$ in diameter, and the cores were obtained from well-preserved, morphologically representative tumour tissue samples in archived, formalin-fixed, paraffin-embedded blocks. The assembled array was held in an X-Y position guide with a 1-mm increment between the individual samples, a 2-mm depth puncture prevention device and semi-automatic micrometers. Considering the limitations associated with obtaining representative areas of a tumour, the present study used duplicate $2.0-\mathrm{mm}$ diameter tissue cores from each donor block. The percentage of tumour in each tissue core was $>70 \%$.

Immunohistochemistry. For IHC staining, the TMA slides were deparaffinised with heat at $55^{\circ} \mathrm{C}$ for $30 \mathrm{~min}$, followed by three 5-min washes with xylene (Duksan Pure Chemicals Co., Ltd., Ansan, Korea). The sections were then rehydrated by a series of successive 5-min washes in 100, 90 and 70\% ethanol (Duksan Pure Chemicals Co., Ltd.). Antigens were retrieved by microwaving the samples for $4 \mathrm{~min} 20 \mathrm{sec}$ in $250 \mathrm{ml}$ of $10 \mathrm{mM}$ sodium citrate (pH 6.0, Duksan Pure Chemicals Co., Ltd.). Furthermore, endogenous peroxidase activity was blocked by incubation with $0.3 \%$ hydrogen peroxidase (Duksan Pure Chemicals Co., Ltd.) for 20 min. Immunostaining for PR, ER, HER2, p53 and Ki-67 was performed using a DakoCytomation Autostainer with a universal staining system and a ChemMate ${ }^{\mathrm{TM}}$ Dako EnVision $^{\text {TM }}$ Detection kit (Dako North America, Inc., Carpinteria, CA, USA). The primary antibodies used were as follows: Anti-PR (1:200 dilution; Dako, Glostrup, Denmark), anti-ER (1:200 dilution; Lab Vision Corporation, Fremont, CA, USA), anti-p53 (1:5,000 dilution; Sigma-Aldrich, St. Louis, MO, USA), anti-HER2 (1:200 dilution; Dako) and anti-Ki-67 (1:200 dilution; Dako). For fascin, affinity-purified rabbit anti-human-fascin polyclonal antibody (1:500 dilution; Leica Microsystems Ltd., Milton Keynes, UK) was used, and detection (4 min incubation at room temperature) was performed with the UltraTech horseradish-peroxidase streptavidin-biotin detection system (Beckman Coulter, Inc., Marseille, France) using an automatic staining machine from the Bond ${ }^{\mathrm{TM}}$ Intense $\mathrm{R}$ Detection kit (Leica Microsystems Ltd., Milton Keynes, UK).

Interpretation of IHC staining. The sections were observed using an Olympus BX51 light microscope (Olympus Corporation, Tokyo, Japan). The IHC results were used to classify the tumours into the following five molecular subtypes: Luminal A (ER-and/or PR-positive, HER2-negative, Ki-67 low), luminal B HER2-positive (ER- and/or PR-positive, HER2-positive), luminal B HER2-negative (ER- and/or PR-positive, HER2-negative, Ki-67 high), HER2-positive (ER- and PR-negative, HER2-positive) and triple-negative (ER-, PR- and HER2-negative) (9,29-31).

Positive fascin immunostaining was defined as exclusive cytoplasmic staining with no nuclear staining. Normal tissue composed of endothelial cells was used as the positive control tissue. Staining intensity of tumour cells was graded on a scale of $0-3$, as follows: No staining, 0 ; weak 1; moderate, 2; or strong, 3 (Fig. 1). In addition, the extent of tumour staining was scored based on the percentage of tumour cells exhibiting staining, using the following scoring system: 1-25\% staining, 1; $26-50 \%$ staining, 2; 51-75\% staining, 3; or 76-100\% staining, 4. In cases with a discrepancy between the duplicated cores, the highest score of the two tissue cores was used as the final score. To calculate the combined immunoreactive score, the staining intensity and extent of tumour staining scores were multiplied $(9,32)$. 
A

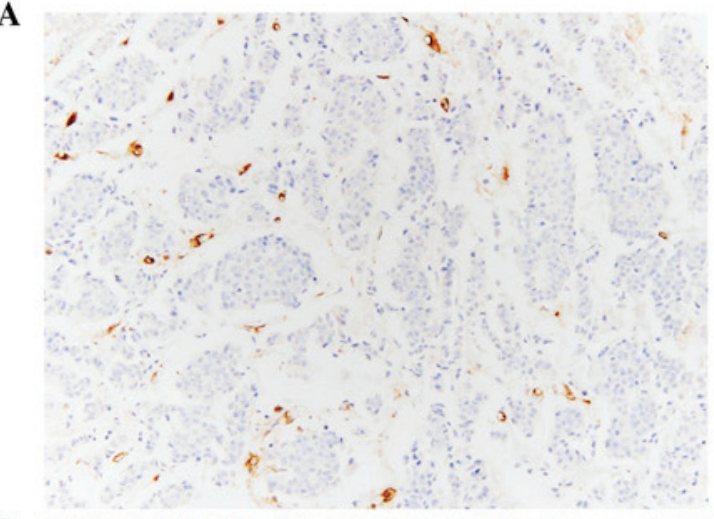

B

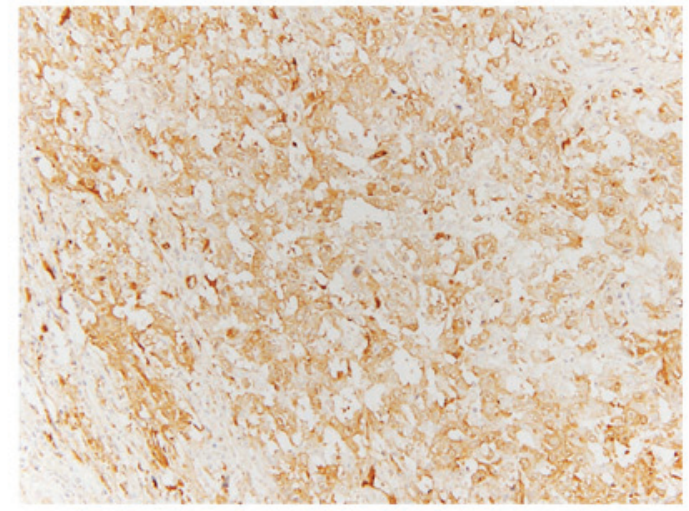

C

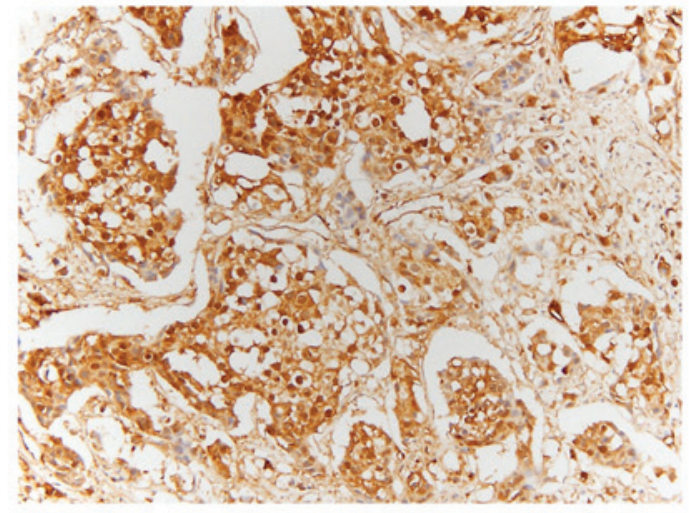

D

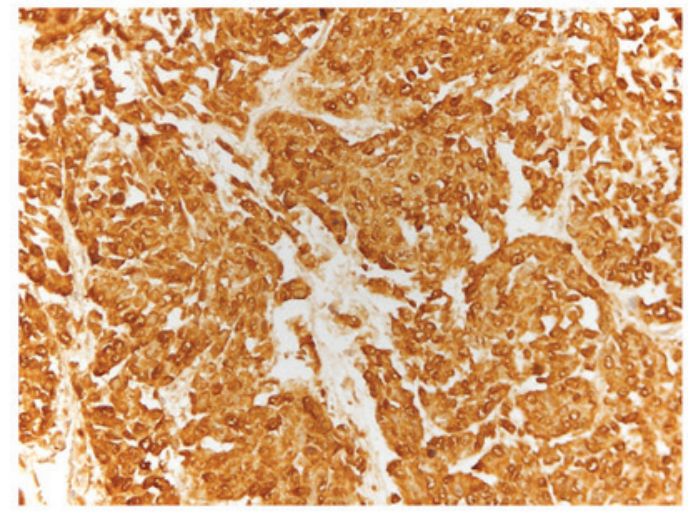

Figure 1. Representative microphotographs revealing (A) negative, (B) weak, (C) moderate and (D) strong intensity fascin expression using immunohistochemical staining (original magnification X200).

The optimal cut-off values for fascin expression were calculated by plotting receiver operating characteristic (ROC) curves of sensitivity versus 1 -specificity. The cut-off value calculated from the ROC curve was then used to evaluate the association between patient mortality and fascin expression.

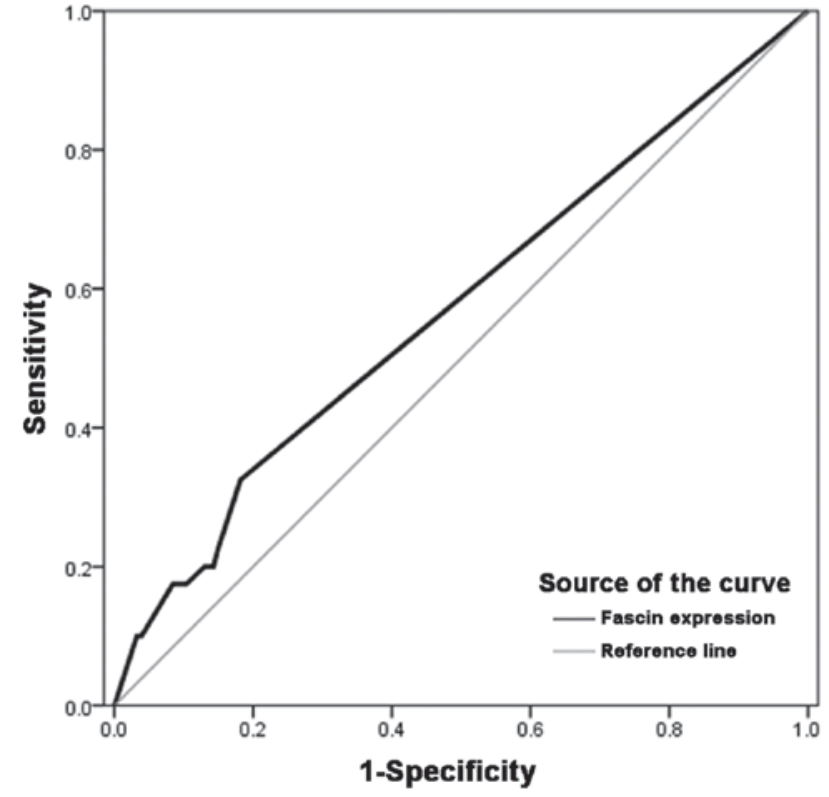

Figure 2. ROC curve for determination of the optimal cut-off value for fascin expression correlated with overall survival rate in patients with invasive ductal carcinoma of the breast (area under the ROC, 0.572). ROC, receiver operating curve.

The ROC curves revealed effective discriminatory power for the correlation between overall survival and fascin expression in the tumour samples (area under the ROC curve, 0.572; Fig. 2). Using the ROC curve, fascin expression was classified as negative (intensity score, $<1$ ) or positive (intensity score, $\geq 1$ ).

Statistical analysis. Correlations between specific clinicopathological parameters and fascin expression were analysed by performing the $\chi^{2}$ test, the linear by linear association test and Fisher's exact test. Furthermore, a Student's t-test was used to examine the association between fascin protein expression and continuous variables, including p53 expression and Ki-67 labelling index. Disease-free survival was defined as the time from the date of diagnosis to the date of recurrence or development of novel distant metastasis. Similarly, overall survival was defined as the time from the date of treatment to the final follow-up visit or cancer-associated mortality. Survival curves were generated using the Kaplan-Meier method and were compared by performing the Tarone-Ware test. Additionally, multivariate analysis was performed to identify independent prognostic markers for disease-free and overall survival using a Cox multistep regression model. $\mathrm{P}<0.05$ was considered to indicate a statistically significant difference, and all statistical analyses were performed using SPSS statistical software for Windows (version 18.0; SPSS Inc., Chicago, IL, USA).

\section{Results}

Patient characteristics. The mean and median ages of the patients were 48.2 and 47.0 years, respectively (range, 26-79 years). Treatment strategies for these cases of breast cancer included modified radical mastectomy with axillary lymph node dissection (151 patients; $77.8 \%$ ), modified 


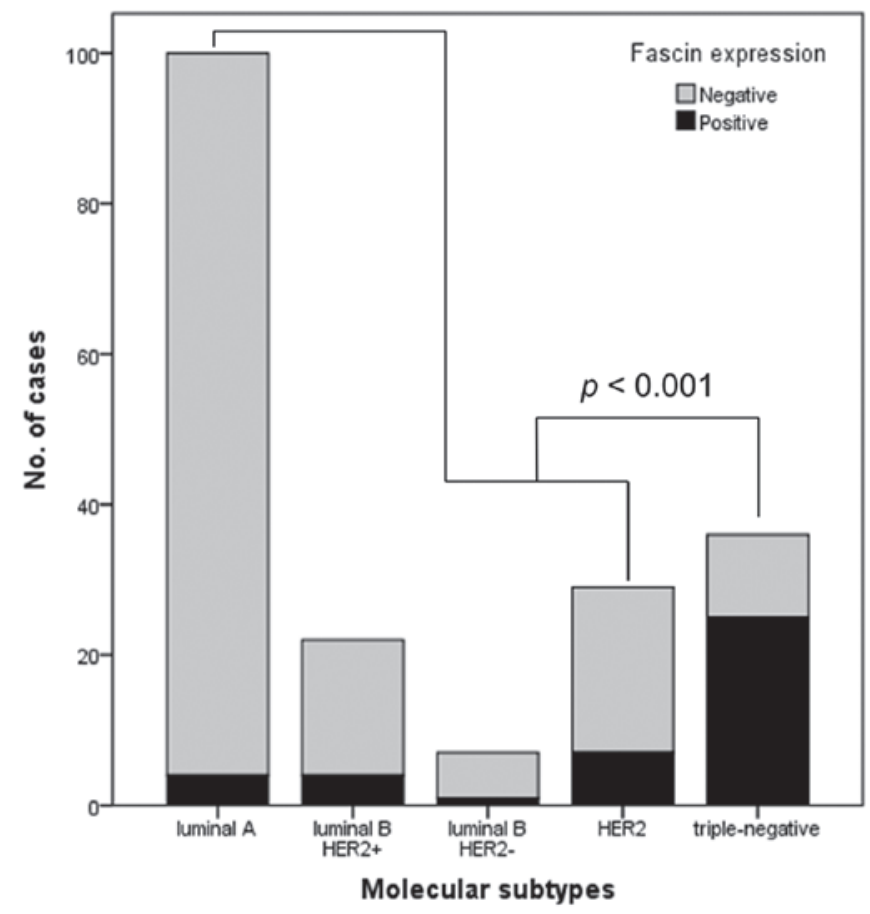

Figure 3. Fascin expression is more frequently observed in triple-negative cases of breast cancer compared with other subtypes $(\mathrm{P}<0.001)$. HER2, human epithelial growth factor receptor 2 .

radical mastectomy (25 patients; $12.9 \%$ ), breast-conserving surgery with axillary lymph node dissection (8 patients; $4.1 \%$ ) and breast-conserving surgery without axillary lymph node dissection (10 patients; $5.2 \%$ ). At the time of surgery, the $\mathrm{T}$ and $\mathrm{N}$ classifications of the current cohort were as follows: T1, 66 patients (34\%); T2, 114 patients $(58.8 \%)$; T3, 14 patients (7.2\%); and N0, 86 patients (44.3\%); N1, 63 patients $(32.5 \%)$; N2, 22 patients $(11.3 \%)$; N3, 23 patients $(11.9 \%)$. Furthermore, the distribution of AJCC staging was as follows: Stage I, 38 patients (19.6\%); stage II, 109 patients (56.2\%); and stage III, 47 patients (24.2\%). Overall, 149 patients received combination chemotherapy with trastuzumab and tamoxifen, 36 received trastuzumab chemotherapy alone and 7 received tamoxifen chemotherapy alone. The remaining 2 patients, who did not receive further treatment, were diagnosed with tubular carcinoma and a 2-mm IDC, respectively. Following surgery, $49(25.3 \%)$ patients developed local recurrence or novel distant metastases, and 40 (20.6\%) patients succumbed during the mean follow-up period of 74.2 months.

Fascin expression is correlated with certain clinicopathological parameters and molecular subtype. In the present study, fascin expression was positive in 41/194 (21.1\%) and negative in 153/194 (78.9\%) cases of IDC. Fascin expression was significantly associated with local recurrence, a high histological grade, tumour necrosis, ER- and PR-negativity, and high expression of p53 and Ki-67 (all $\mathrm{P}<0.05$ ), but was not significantly associated with HER2 positivity (Table I). Notably, fascin expression was significantly correlated with resistance to adjuvant therapy $(\mathrm{P}<0.001)$.

Compared with the other subtypes investigated, triple-negative breast cancer was associated with old age and vascular invasion. HER2 and triple-negative breast cancer were frequently observed in patients with a high histological grade and tumour necrosis (all $\mathrm{P}<0.05$; data not shown). Furthermore, luminal B HER2-negative and HER2 breast cancer were correlated with lymphatic invasion, and patients with luminal B HER2-negative breast cancer exhibited a good response to adjuvant therapy compared with that of other subtypes (all $\mathrm{P}<0.05$; data not shown).

Fascin expression is associated with triple-negative breast cancer and correlated with patient survival. With respect to molecular subtype, the distribution of fascin expression was as follows: Four cases of luminal A (4\%), 1 case of luminal B HER2-negative (14.3\%), 4 cases of luminal B HER2-positive (18.2\%), 25 cases of triple-negative $(69.4 \%)$ and 7 cases of HER 2 (24.1\%) breast cancer. A comparison of the various molecular subtypes revealed significantly greater occurrence of fascin expression in the triple-negative subtype than in the luminal A, luminal B or HER2 subtypes ( $\mathrm{P}<0.001$; Fig. 3$)$.

Analysis of patient survival using the Kaplan-Meier method identified that fascin expression was significantly correlated with poor disease-free and overall survival rates $(\mathrm{P}<0.05$; Fig. 4). Other prognostic factors, including advanced tumour stage, high histological grade and lymphatic and perineural invasions, were also correlated with worse disease-free and overall survival in univariate analysis (all $\mathrm{P}<0.05$; Table II). In multivariate analyses, the significant correlation between fascin and overall survival persisted $(\mathrm{P}=0.013)$, whereas fascin expression only exhibited a marginal association with decreased disease-free survival $(\mathrm{P}<0.069)$.

Fascin expression is correlated with patient survival, according to AJCC TNM staging and response to adjuvant therapy. Fascin expression was associated with poor disease-free and overall survival in patients with an advanced tumour stage, nodal metastasis or advanced AJCC stage (all $\mathrm{P}<0.05$; Fig. 5). However, no significant correlation was observed between fascin and survival rate for patients with early stage disease. In multivariate analyses, the significance of the association between fascin expression and disease-free or overall survival was retained for patients with an advanced AJCC stage, and the association between fascin expression and disease-free survival was retained for patients with an advanced $\mathrm{T}$ classification (all $\mathrm{P}<0.05$; Table III).

Analysis according to the adjuvant treatment response, identified that fascin expression was significantly associated with disease-free and overall survival in patients with resistance to adjuvant therapy, however, no significance was observed following adjustment for the aforementioned potential confounders (data not shown).

\section{Discussion}

During cancer progression, an increase in cell motility is essential for tumour invasion and subsequent dissemination or metastasis. This increase in motility occurs via the modulation of actin filaments to form finger-like plasma membrane protrusions termed invadopodia, $(33,34)$. Such dynamic rearrangement of the actin cytoskeleton is regulated by numerous actin-binding proteins (35), including fascin. 
$\mathbf{A}$

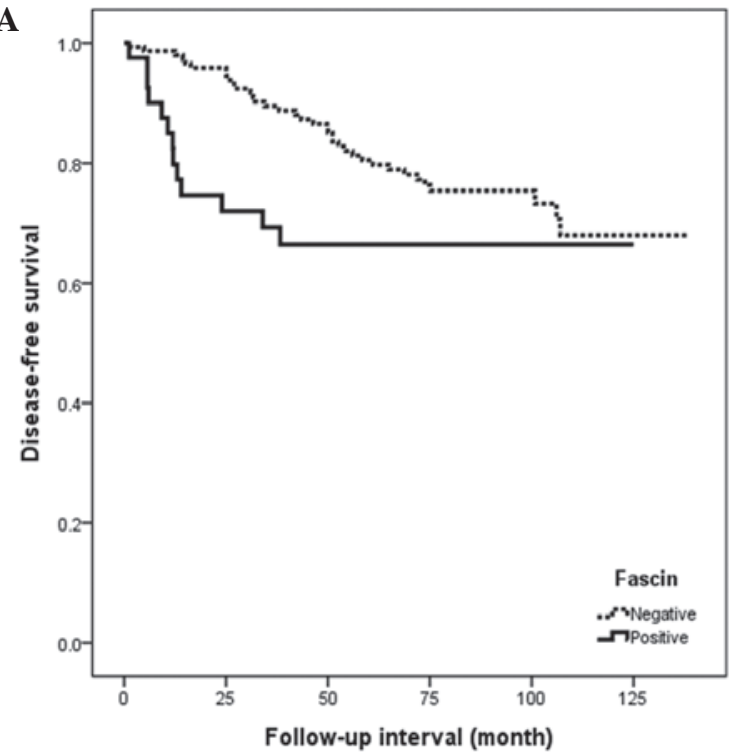

B

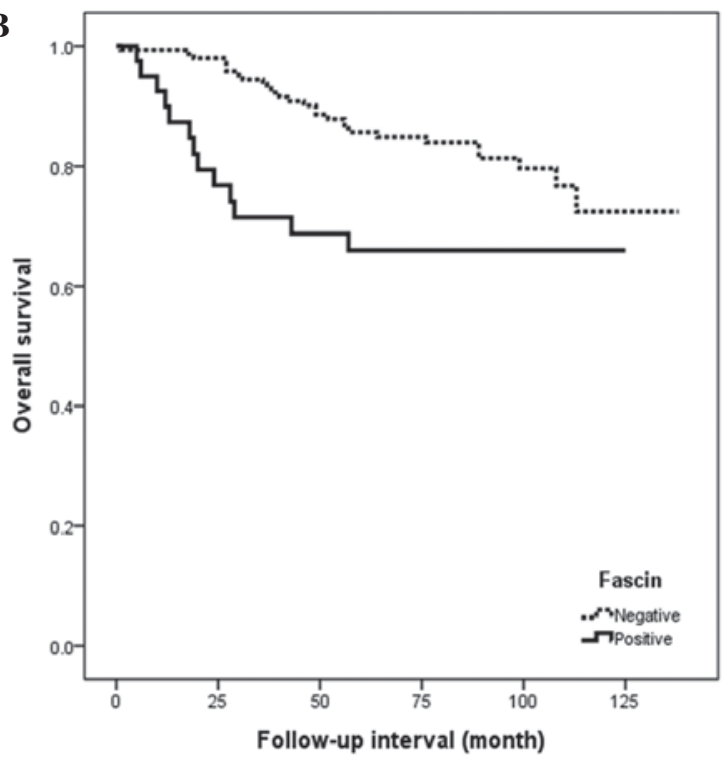

Figure 4. Fascin expression is correlated with poor disease-free and overall survival rates in patients with invasive duct carcinoma of the breast. (A) Disease-free and (B) overall survival curves derived using the Kaplan-Meier method, demonstrating significant correlation with fascin expression. Disease-free survival, $\mathrm{P}=0.045$; overall survival, $\mathrm{P}=0.004$.

A

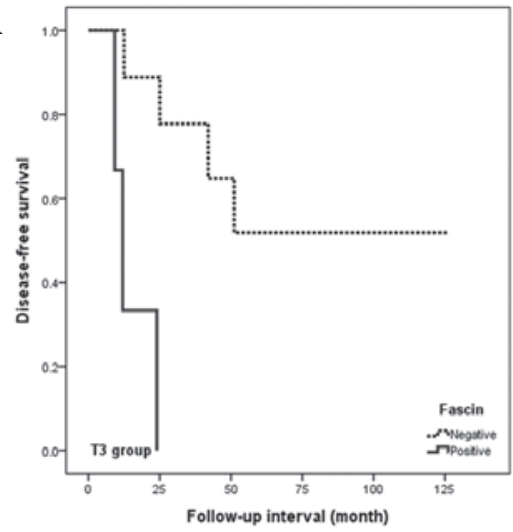

B

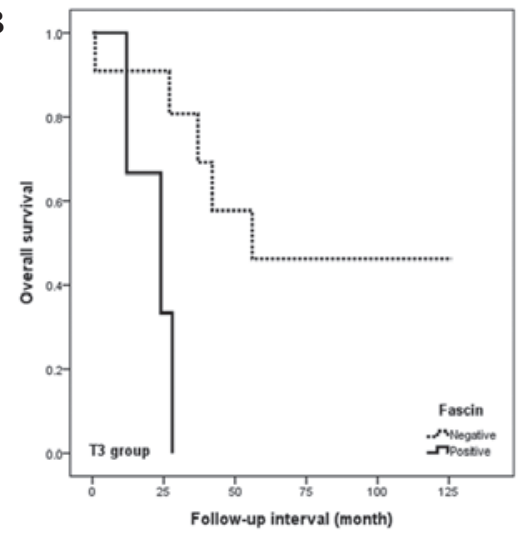

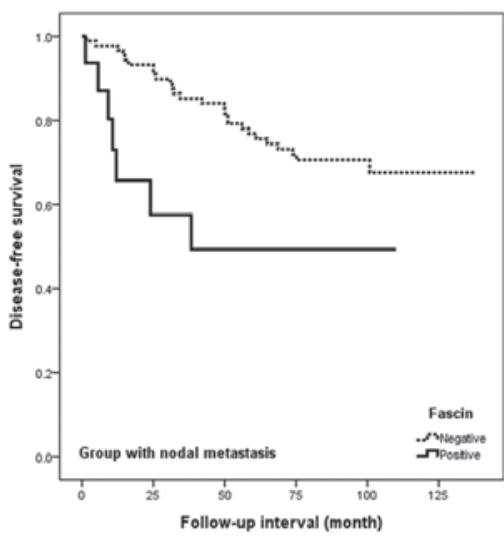
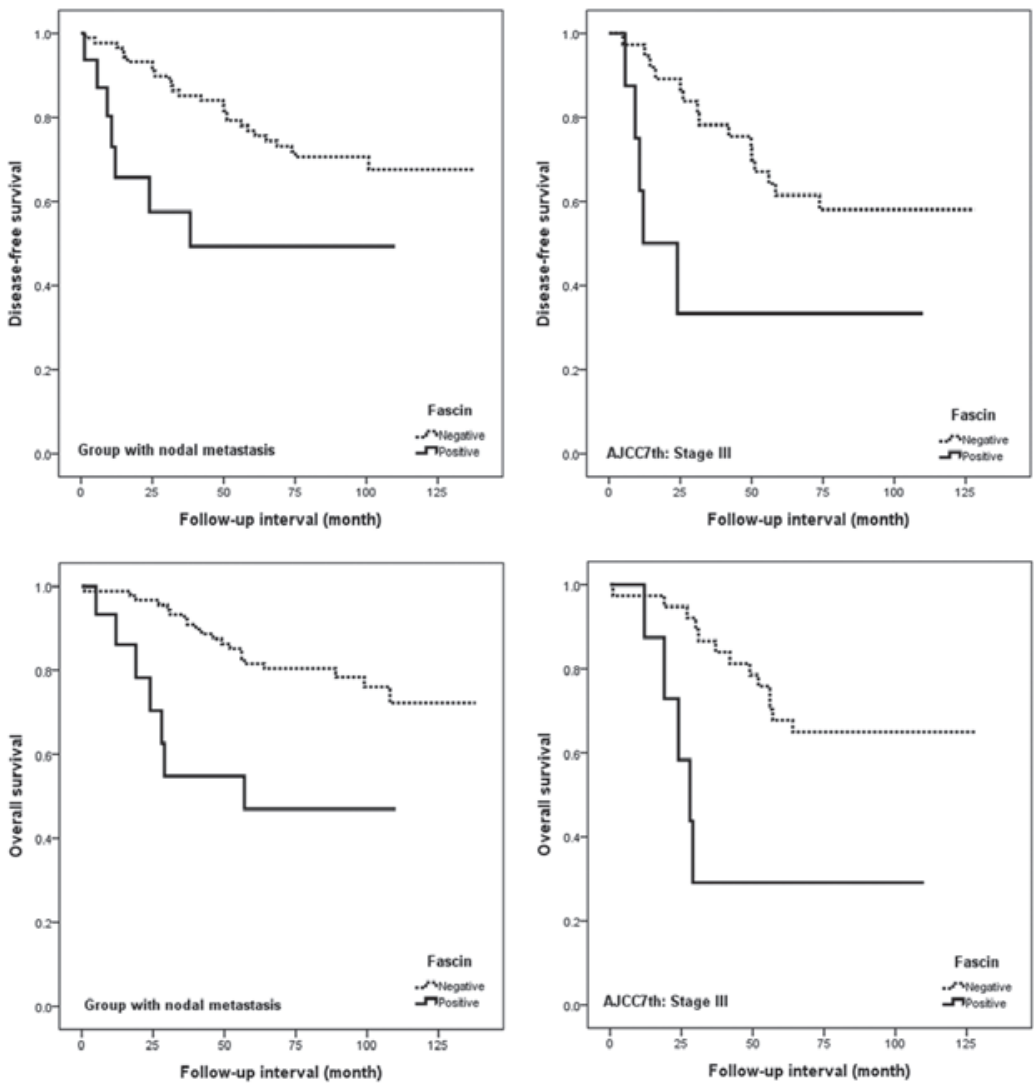

Figure 5. Fascin expression is correlated with disease-free and overall survival rates, according to T3, nodal metastasis and AJCC stage III in invasive duct carcinoma of the breast. (A) Disease-free and (B) overall survival curves derived using the Kaplan-Meier method, demonstrating significant correlation with fascin expression according to T3, nodal metastasis and AJCC stage III advanced breast cancer. Disease-free survival, P=0.001, 0.009 and 0.007; overall survival $\mathrm{P}=0.013,0.001$ and 0.004 , respectively. AJCC, American Joint Committee on Cancer.

Fascin is an actin cross-linking protein, which localises to filopodia at the leading edge of migratory cells. Enhanced fascin expression is associated with increased cell migration and invasion (36). Studies have demonstrated that fascin expression is significantly associated with triple-negativity, as well as poor clinical outcome, in hormone receptor-negative or triple-negative breast cancer $(25,26)$. However, to the best of our knowledge, no previous studies have, thus 
Table I. Correlation between clinicopathological parameters and fascin expression in invasive ductal carcinoma.

\begin{tabular}{|c|c|c|c|c|}
\hline \multirow[b]{2}{*}{ Parameter } & \multirow[b]{2}{*}{ Patients, n $(n=194)$} & \multicolumn{2}{|c|}{ Fascin expression, $\mathrm{n}(\%)$} & \multirow[b]{2}{*}{ P-value } \\
\hline & & Negative $(n=153)$ & Positive $(n=41)$ & \\
\hline \multicolumn{5}{|l|}{ Age, years } \\
\hline$\leq 47$ & 104 & $82(53.6)$ & $22(53.7)$ & $0.994^{\mathrm{a}}$ \\
\hline$>47$ & 90 & $71(46.4)$ & $19(46.3)$ & \\
\hline \multicolumn{5}{|l|}{ AJCC stage } \\
\hline I & 38 & $29(19.0)$ & $9(22.0)$ & $0.441^{\mathrm{b}}$ \\
\hline II & 109 & $85(55.6)$ & $24(58.5)$ & \\
\hline III & 47 & $39(25.5)$ & $8(19.5)$ & \\
\hline \multicolumn{5}{|l|}{ T category } \\
\hline $\mathrm{T} 1$ & 66 & $55(35.9)$ & $11(26.8)$ & $0.369^{\mathrm{b}}$ \\
\hline $\mathrm{T} 2$ & 114 & $87(56.9)$ & $27(65.9)$ & \\
\hline $\mathrm{T} 3$ & 14 & $11(7.2)$ & $3(7.3)$ & \\
\hline \multicolumn{5}{|l|}{$\mathrm{N}$ category } \\
\hline N0 & 86 & $61(39.9)$ & $25(61.0)$ & $0.155^{\mathrm{b}}$ \\
\hline $\mathrm{N} 1$ & 63 & $54(35.3)$ & $9(22.0)$ & \\
\hline $\mathrm{N} 2$ & 22 & $21(13.7)$ & $1(2.4)$ & \\
\hline N3 & 23 & $17(11.1)$ & $6(14.6)$ & \\
\hline \multicolumn{5}{|c|}{ Local recurrence } \\
\hline Absence & 188 & $151(98.7)$ & $37(90.2)$ & $0.019^{\mathrm{c}, \mathrm{d}}$ \\
\hline Presence & 6 & $2(1.3)$ & $4(9.8)$ & \\
\hline \multicolumn{5}{|c|}{ Distant metastasis } \\
\hline Absence & 147 & $118(77.1)$ & $29(70.7)$ & $0.396^{\mathrm{a}}$ \\
\hline Presence & 47 & $35(22.9)$ & $12(29.3)$ & \\
\hline \multicolumn{5}{|c|}{ Tumour size, $\mathrm{cm}$} \\
\hline$\leq 2$ & 69 & $58(37.9)$ & $11(26.8)$ & $0.188^{\mathrm{a}}$ \\
\hline$>2$ & 125 & $95(62.1)$ & $30(73.2)$ & \\
\hline \multicolumn{5}{|l|}{ Tumour border } \\
\hline Well-defined & 40 & $30(19.6)$ & $10(24.4)$ & $0.501^{\mathrm{a}}$ \\
\hline Ill-defined & 154 & $123(80.4)$ & $31(75.6)$ & \\
\hline \multicolumn{5}{|c|}{ Number of tumours } \\
\hline Single & 181 & $142(92.8)$ & $39(95.1)$ & $0.599^{a}$ \\
\hline Multiple & 13 & $11(7.2)$ & $2(4.9)$ & \\
\hline \multicolumn{5}{|l|}{ Paget's disease } \\
\hline Absence & 187 & $146(95.4)$ & $41(100)$ & $0.349^{\mathrm{a}}$ \\
\hline Presence & 7 & $7(4.6)$ & $0(0.0)$ & \\
\hline \multicolumn{5}{|c|}{ Histological grade } \\
\hline 1 & 27 & $26(17.0)$ & $1(2.4)$ & $<0.001^{\mathrm{b}, \mathrm{d}}$ \\
\hline 2 & 89 & $76(49.7)$ & $13(31.7)$ & \\
\hline 3 & 78 & $51(33.3)$ & $27(65.9)$ & \\
\hline \multicolumn{5}{|c|}{ Lymphatic invasion } \\
\hline Negative & 91 & $72(47.1)$ & $19(46.3)$ & $0.935^{\mathrm{a}}$ \\
\hline Positive & 103 & $81(52.9)$ & $22(53.7)$ & \\
\hline \multicolumn{5}{|c|}{ Vascular invasion } \\
\hline Negative & 179 & $144(94.1)$ & $35(85.4)$ & $0.093^{\mathrm{c}}$ \\
\hline Positive & 15 & $9(5.9)$ & $6(14.6)$ & \\
\hline \multicolumn{5}{|c|}{ Perineural invasion } \\
\hline Negative & 163 & $127(83.0)$ & $36(87.8)$ & $0.456^{\mathrm{a}}$ \\
\hline Positive & 31 & $26(17.0)$ & $5(12.2)$ & \\
\hline \multicolumn{5}{|c|}{ Tumour necrosis } \\
\hline Absence & 109 & $95(62.1)$ & $14(34.1)$ & $0.001^{\mathrm{d}}$ \\
\hline Presence & 85 & $58(37.9)$ & $27(65.9)$ & \\
\hline
\end{tabular}


Table I. Continued.

Fascin expression in tumour, $\mathrm{n}(\%)$

\begin{tabular}{|c|c|c|c|c|}
\hline Parameter & Patients, n $(n=194)$ & Negative $(\mathrm{n}=153)$ & Positive $(n=41)$ & P-value ${ }^{a}$ \\
\hline \multicolumn{5}{|l|}{ Central tumour fibrosis } \\
\hline Absence & 158 & $122(79.7)$ & $36(87.8)$ & $0.238^{\mathrm{a}}$ \\
\hline Presence & 36 & $31(20.3)$ & $5(12.2)$ & \\
\hline \multicolumn{5}{|l|}{ EIC } \\
\hline Absence & 163 & $125(81.7)$ & $38(92.7)$ & $0.088^{\mathrm{a}}$ \\
\hline Presence & 31 & $28(18.3)$ & $3(7.3)$ & \\
\hline \multicolumn{5}{|l|}{ Oestrogen receptor } \\
\hline Negative & 65 & $33(21.6)$ & $32(78)$ & $<0.001^{\mathrm{f}}$ \\
\hline Positive & 129 & $120(78.4)$ & $9(22)$ & \\
\hline \multicolumn{5}{|l|}{ Progesterone receptor } \\
\hline Negative & 92 & $56(36.6)$ & $36(87.8)$ & $<0.001^{\mathrm{f}}$ \\
\hline Positive & 102 & $97(63.4)$ & $5(12.2)$ & \\
\hline \multicolumn{5}{|l|}{ HER2 } \\
\hline Negative & 143 & $113(73.9)$ & $30(73.2)$ & $0.929^{\mathrm{a}}$ \\
\hline Positive & 51 & $40(26.1)$ & $11(26.8)$ & \\
\hline \multicolumn{5}{|l|}{ Adjuvant therapy ${ }^{\mathrm{f}}$} \\
\hline Responder & 175 & $145(96)$ & $30(73.2)$ & $<0.001^{\mathrm{d}}$ \\
\hline Non-responder & 17 & $6(4.0)$ & $11(26.8)$ & \\
\hline p53 expression & & 20.84 & 63.33 & $<0.001^{\mathrm{d}, \mathrm{e}}$ \\
\hline Ki-67 labelling index & & 2.56 & 7.37 & $<0.001^{\mathrm{d}, \mathrm{e}}$ \\
\hline
\end{tabular}

${ }^{\mathrm{a}} \chi^{2}$ test; ${ }^{\mathrm{b}}$ linear by linear association test; ${ }^{\mathrm{c}} \mathrm{Fisher}$ 's exact test; ${ }^{\mathrm{d}} \mathrm{P}<0.05$; ${ }^{\mathrm{e}} \mathrm{Student}$ 's $\mathrm{t}$ test; ${ }^{\mathrm{f}}$ excludes two patients that did not undergo adjuvant therapy. AJCC, American Joint Committee on Cancer; EIC, extensive intraductal component; HER2, human epidermal growth factor receptor 2.

Table II. Disease-free and overall survival analyses $(n=194)$.

\begin{tabular}{|c|c|c|c|c|}
\hline Survival & $\begin{array}{c}\text { Univariate } \\
\text { significance }^{\mathrm{a}}\end{array}$ & $\begin{array}{l}\text { Multivariate } \\
\text { significance }^{\mathrm{b}}\end{array}$ & Hazard ratio & $95 \% \mathrm{CI}$ \\
\hline \multicolumn{5}{|l|}{ Disease-free } \\
\hline Fascin expression (negative vs. positive) & 0.045 & 0.069 & 1.878 & $0.952-3.705$ \\
\hline AJCC stage (I or II vs. III) & $<0.001^{\mathrm{c}}$ & $0.047^{\mathrm{c}}$ & 1.898 & $1.01-3.568$ \\
\hline Histological grade ( 1 or 2 vs. 3 ) & $0.003^{\mathrm{c}}$ & 0.182 & 1.555 & $0.813-2.975$ \\
\hline Lymphatic invasion (absence vs. presence) & $0.002^{\mathrm{c}}$ & 0.206 & 1.600 & $0.772-3.316$ \\
\hline Perineural invasion (absence vs. presence) & $<0.001^{\mathrm{c}}$ & $<0.001^{\mathrm{c}}$ & 3.594 & $1.92-6.726$ \\
\hline \multicolumn{5}{|l|}{ Overall } \\
\hline Fascin expression (negative vs. positive) & $0.004^{\mathrm{c}}$ & 0.013 & 2.475 & $1.214-5.042$ \\
\hline AJCC stage (I or II vs. III) & $<0.001$ & 0.059 & 1.954 & $0.976-3.912$ \\
\hline Histological grade (1 or 2 vs. 3 ) & $<0.001^{\mathrm{c}}$ & 0.205 & 1.616 & $0.769-3.393$ \\
\hline Lymphatic invasion (absence vs. presence) & $0.001^{\mathrm{c}}$ & 0.127 & 1.936 & $0.829-4.519$ \\
\hline Perineural invasion (absence vs. presence) & $<0.001^{\mathrm{c}}$ & $0.001^{\mathrm{c}}$ & 3.148 & $1.577-6.283$ \\
\hline
\end{tabular}

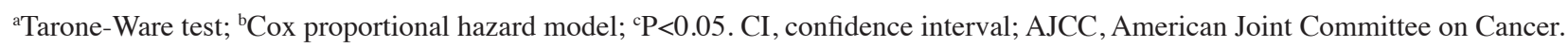

far, identified an association between fascin expression and survival in patients with late-stage disease or resistance to adjuvant therapy.

Previous studies have demonstrated that fascin expression is associated with reduced survival in various types of cancer $(20-23,26,37)$. The expression of fascin in tumours was initially considered to be important in promoting tumour progression, and numerous studies of colon cancer have proposed that fascin is more important in advanced stage disease compared with early stage disease $(20,37,38)$. 
Table III. Correlations between disease-free and overall survival, and fascin expression according to the size and/or extent of the tumour, nodal metastasis and AJCC stage group.

A, Disease-free survival

\begin{tabular}{|c|c|c|c|c|c|}
\hline Survival & Recurrence/total & $\begin{array}{l}\text { Univariate } \\
\text { significance }^{a}\end{array}$ & $\begin{array}{l}\text { Multivariate } \\
\text { significance }^{b}\end{array}$ & Hazard ratio & $95 \% \mathrm{CI}$ \\
\hline \multicolumn{6}{|l|}{ T stage } \\
\hline $\mathrm{T} 1$ & $14 / 66$ & 0.969 & 0.502 & 1.757 & $0.338-9.126$ \\
\hline $\mathrm{T} 2$ & $28 / 114$ & 0.177 & 0.491 & 1.363 & $0.565-3.291$ \\
\hline $\mathrm{T} 3$ & $7 / 14$ & $0.001^{\mathrm{c}}$ & $0.044^{\mathrm{c}}$ & 15.512 & $1.08-222.759$ \\
\hline \multicolumn{6}{|c|}{ Nodal metastasis } \\
\hline No & $16 / 86$ & 0.217 & 0.456 & 1.511 & $0.51-4.479$ \\
\hline Yes & $33 / 108$ & $0.009^{c}$ & 0.186 & 1.842 & $0.745-4.556$ \\
\hline \multicolumn{6}{|c|}{ AJCC stage } \\
\hline I & $7 / 38$ & 0.605 & 0.491 & 1.920 & $0.301-12.26$ \\
\hline II & $22 / 109$ & 0.203 & 0.661 & 1.251 & $0.459-3.41$ \\
\hline III & $20 / 47$ & $0.007^{\mathrm{c}}$ & $0.029^{c}$ & 3.800 & $1.148-12.58$ \\
\hline
\end{tabular}

B, Overall survival

\begin{tabular}{|c|c|c|c|c|c|}
\hline Survival & Mortality/total & $\begin{array}{l}\text { Univariate } \\
\text { significance }^{\mathrm{a}}\end{array}$ & $\begin{array}{l}\text { Multivariate } \\
\text { significance }^{b}\end{array}$ & Hazard ratio & $95 \%$ CI \\
\hline \multicolumn{6}{|l|}{ T stage } \\
\hline $\mathrm{T} 1$ & $12 / 66$ & 0.701 & 0.283 & 2.562 & $0.461-14.245$ \\
\hline $\mathrm{T} 2$ & $20 / 114$ & $0.013^{\mathrm{c}}$ & 0.065 & 2.551 & $0.942-6.904$ \\
\hline $\mathrm{T} 3$ & $8 / 14$ & $0.013^{\mathrm{c}}$ & 0.067 & 9.952 & $0.848-116.797$ \\
\hline \multicolumn{6}{|c|}{ Nodal metastasis } \\
\hline No & $13 / 86$ & 0.660 & 0.204 & 2.143 & $0.66-6.958$ \\
\hline Yes & $27 / 108$ & $0.001^{\mathrm{c}}$ & 0.101 & 2.185 & $0.858-5.566$ \\
\hline \multicolumn{6}{|c|}{ AJCC stage } \\
\hline I & $5 / 38$ & 0.361 & 0.507 & 1.880 & $0.292-12.125$ \\
\hline II & $17 / 109$ & 0.024 & 0.098 & 2.554 & 0.84-7.767 \\
\hline III & $18 / 47$ & $0.004^{\mathrm{c}}$ & $0.048^{c}$ & 3.239 & $1.012-10.366$ \\
\hline
\end{tabular}

${ }^{a}$ Tarone-Ware test and ${ }^{\mathrm{b}} \mathrm{Cox}$ proportional hazard model, adjusted for histological grade, and lymphatic and perineural invasion. ${ }^{\mathrm{c}} \mathrm{P}<0.05$. AJCC, American Joint Committee on Cancer; CI, confidence interval.

However, a study based on lung cancer reported that fascin expression may be associated with shorter survival in patients with early stage disease (22), although an alternative study of lung cancer did not observe a significant association between fascin expression and survival in early stage disease (39). Thus, there is controversy regarding the association between fascin expression and the clinical outcomes of patients with cancer. In the present study, fascin expression was significantly associated with poor disease-free and overall survival in patients with advanced stage breast cancer. Additionally, fascin expression was significantly associated with worse disease-free and overall survival in patients who were resistant to adjuvant therapy in univariate analyses, although this significance did not remain following multivariate analyses.

Fascin may enhance tumour cell motility and invasion in addition to accelerating tumour cell proliferation, thereby enhancing cancer cell survival and contributing to the development of additional distant metastases $(40,41)$. In the present study, fascin expression was significantly associated with local recurrence and the survival of patients with late stage disease. This may be due to the role of fascin in upregulating other proteins known to be critical for the execution of metastasis, for example urokinase-type plasminogen activator, and matrix metalloproteinases-2 and -9 (19). However, the precise mechanisms by which fascin promotes cancer development and progression are not fully understood, and the potential prognostic value of fascin may vary according to biological factors, the degree of cancer progression and therapeutic tolerance.

There were a number of limitations to the present study. Firstly, it shares similarities with a previous retrospective study that did not identify continuous associations over time (42). Therefore, the current results did not clarify whether associations with fascin are maintained throughout invasive 
tumour growth or whether they contribute only to the initiation of invasive growth. This is a significant consideration when determining therapeutic strategies based on the control of fascin activity. In addition, the number of patients with late-stage disease or resistance to adjuvant therapy was relatively small, potentially limiting the statistical power of the study in future analyses.

In conclusion, the results of the present study demonstrated that fascin expression was significantly correlated with breast cancer patient survival, particularly for those with late stage disease. In addition, fascin expression was significantly correlated with known predictors of poor survival, including high histological grade, tumour necrosis, ER- and PR-negativity, resistance to adjuvant therapy and elevated expression of p53 and Ki-67. Thus, fascin may be considered as a key protein in the promotion of tumour progression, and may facilitate the prediction of outcomes and improvement of prognostic models in breast cancer. Furthermore, fascin may present a promising therapeutic target for the inhibition of metastasis, particularly in patients with advanced breast cancer.

\section{References}

1. National Cancer Institute: Cancer statistics: SEER stat fact sheets, breast cancer. http://seer.cancer.gov/statfacts/html/breast.html.

2. Bouchalova P, Nenutil R, Muller P, et al: Mutant p53 accumulation in human breast cancer is not an intrinsic property or dependent on structural or functional disruption but is regulated by exogenous stress and receptor status. J Pathol 233: 238-246, 2014.

3. Harris L, Fritsche H, Mennel R, et al: American Society of Clinical Oncology 2007 update of recommendations for the use of tumor markers in breast cancer. J Clin Oncol 25: 5287-5312, 2007.

4. Lin Q, Liu Y, Chen H, et al: Survivin, Ki-67 and tumor grade as predictors of response to docetaxel-based neoadjuvant chemotherapy in locally advanced breast cancer. Mol Clin Oncol 1: 839-844, 2013

5. Mason BH, Holdaway IM, Mullins PR, Yee LH and Kay RG: Progesterone and estrogen receptors as prognostic variables in breast cancer. Cancer Res 43: 2985-2990, 1983.

6. Ogston KN, Miller ID, Payne S, et al: A new histological grading system to assess response of breast cancers to primary chemotherapy: Prognostic significance and survival. Breast 12: 320-327, 2003.

7. Peppercorn J, Perou CM and Carey LA: Molecular subtypes in breast cancer evaluation and management: Divide and conquer. Cancer Invest 26: 1-10, 2008

8. Feeley LP, Mulligan AM, Pinnaduwage D, Bull SB and Andrulis IL: Distinguishing luminal breast cancer subtypes by Ki67, progesterone receptor or TP53 status provides prognostic information. Mod Pathol 27: 554-561, 2014.

9. Chambers AF, Naumov GN, Varghese HJ, et al: Critical steps in hematogenous metastasis: An overview. Surg Oncol Clin N Am 10: 243-255, 2001.

10. Saaristo A, Karpanen T and Alitalo K: Mechanisms of angiogenesis and their use in the inhibition of tumor growth and metastasis. Oncogene 19: 6122-6129, 2000.

11. Woodhouse EC, Chuaqui RF and Liotta LA: General mechanisms of metastasis. Cancer 80 (Suppl): S1529-S1537, 1997.

12. Min KW, Kim DH, Do SI, et al: Diagnostic and prognostic relevance of MMP-11 expression in the stromal fibroblast-like cells adjacent to invasive ductal carcinoma of the breast. Ann Surg Oncol 20 (Suppl 3): S433-S442, 2013.

13. Jiang P, Enomoto A and Takahashi M: Cell biology of the movement of breast cancer cells: Intracellular signalling and the actin cytoskeleton. Cancer Lett 284: 122-130, 2009.

14. Edwards RA and Bryan J: Fascins, a family of actin bundling proteins. Cell Motil Cytoskeleton 32: 1-9, 1995.

15. Mosialos G, Yamashiro S, Baughman RW, et al: Epstein-Barr virus infection induces expression in B lymphocytes of a novel gene encoding an evolutionarily conserved 55-kilodalton actin-bundling protein. J Virol 68: 7320-7328, 1994.
16. Hashimoto Y, Skacel M and Adams JC: Roles of fascin in human carcinoma motility and signaling: Prospects for a novel biomarker? Int J Biochem Cell Biol 37: 1787-1804, 2005.

17. Hsu KF, Lin CK, Yu CP, et al: Cortactin, fascin, and survivin expression associated with clinicopathological parameters in esophageal squamous cell carcinoma. Dis Esophagus 22: 402-408, 2009.

18. Al-Alwan M, Olabi S, Ghebeh $\mathrm{H}$, et al: Fascin is a key regulator of breast cancer invasion that acts via the modification of metastasis-associated molecules. PLoS One 6: e27339, 2011.

19. Hashimoto Y, Skacel M, Lavery IC, et al: Prognostic significance of fascin expression in advanced colorectal cancer: An immunohistochemical study of colorectal adenomas and adenocarcinomas. BMC Cancer 6: 241, 2006.

20. Pelosi G, Pastorino U, Pasini F, et al: Independent prognostic value of fascin immunoreactivity in stage I nonsmall cell lung cancer. Br J Cancer 88: 537-547, 2003.

21. Hashimoto Y, Shimada Y, Kawamura J, et al: The prognostic relevance of fascin expression in human gastric carcinoma. Oncology 67: 262-270, 2004.

22. Tong GX, Yee H, Chiriboga L, Hernandez O and Waisman J: Fascin-1 expression in papillary and invasive urothelial carcinomas of the urinary bladder. Hum Pathol 36: 741-746, 2005.

23. Tan VY, Lewis SJ, Adams JC and Martin RM: Association of fascin-1 with mortality, disease progression and metastasis in carcinomas: A systematic review and meta-analysis. BMC Med 11: 52, 2013.

24. Grothey A, Hashizume R, Sahin AA and McCrea PD: Fascin, an actin-bundling protein associated with cell motility, is upregulated in hormone receptor negative breast cancer. Br J Cancer 83: 870-873, 2000.

25. Yoder BJ, Tso E, Skacel M, et al: The expression of fascin, an actin-bundling motility protein, correlates with hormone receptor-negative breast cancer and a more aggressive clinical course. Clin Cancer Res 11: 186-192, 2005.

26. Esnakula AK, Ricks-Santi L, Kwagyan J, et al: Strong association of fascin expression with triple negative breast cancer and basal-like phenotype in African-American women. J Clin Pathol 67: 153-160, 2014.

27. Robbins P, Pinder S, de Klerk N, et al: Histological grading of breast carcinomas: A study of interobserver agreement. Hum Pathol 26: 873-879, 1995.

28. Edge SB, Byrd DR, Compton CC, et al (eds). AJCC Cancer Staging Manual. 7th edition. Springer, New York, NY, USA, 2010.

29. Allred DC, Harvey JM, Berardo M and Clark GM: Prognostic and predictive factors in breast cancer by immunohistochemical analysis. Mod Pathol 11: 155-168, 1998.

30. Carey LA, Perou CM, Livasy CA, et al: Race, breast cancer subtypes, and survival in the Carolina Breast Cancer Study. JAMA 295: 2492-2502, 2006.

31. Wolff AC, Hammond ME, Hicks DG, et al; American Society of Clinical Oncology; College of American Pathologists: Recommendations for human epidermal growth factor receptor 2 testing in breast cancer: American Society of Clinical Oncology/College of American Pathologists clinical practice guideline update. J Clin Oncol 31: 3997-4013, 2013.

32. Remmele W and Stegner HE: Recommendation for uniform definition of an immunoreactive score (IRS) for immunohistochemical estrogen receptor detection (ER-ICA) in breast cancer tissue. Pathologe 8: 138-140, 1987.

33. Jayo A and Parsons M: Fascin: A key regulator of cytoskeletal dynamics. Int J Biochem Cell Biol 42: 1614-1617, 2010.

34. Li A, Dawson JC, Forero-Vargas M, et al: The actin-bundling protein fascin stabilizes actin in invadopodia and potentiates protrusive invasion. Curr Biol 20: 339-345, 2010.

35. Matsudaira P: Actin crosslinking proteins at the leading edge. Semin Cell Biol 5: 165-174, 1994.

36. Vignjevic D, Schoumacher M, Gavert N, et al: Fascin, a novel target of beta-catenin-TCF signaling, is expressed at the invasive front of human colon cancer. Cancer Res 67: 6844-6853, 2007.

37. Oh SY, Kim YB, Suh KW, et al: Prognostic impact of fascin-1 expression is more significant in advanced colorectal cancer. J Surg Res 172: 102-108, 2012.

38. Puppa G, Maisonneuve P, Sonzogni A, et al: Independent prognostic value of fascin immunoreactivity in stage III-IV colonic adenocarcinoma. Br J Cancer 96: 1118-1126, 2007.

39. Roh MS, Um SJ, Choi Y, et al: Prognostic significance of fascin expression in stage I non-small cell lung cancer. Tuberc Respir Dis (Seoul) 65: 105-109, 2008. 
40. Xing P, Li JG, Jin F, et al: Fascin, an actin-bundling protein, promotes breast cancer progression in vitro. Cell Biochem Funct 29: 303-310, 2011.

41. Xie JJ, Xu LY, Wu JY, et al: Involvement of CYR61 and CTGF in the fascin-mediated proliferation and invasiveness of esophageal squamous cell carcinomas cells. Am J Pathol 176: 939-951, 2010.
42. Gu MJ, Kim JY and Park JB: Fascin expression predicts lymph node metastasis and worse survival in small intestinal carcinoma. Pathology 46: 21-24, 2014. 